the first week of treatment. In 1 case the pain continued during the whole stay in hospital.

Haberman' reported that, in 1906, 135 cases had been treated by Professor Lenhartz in Hamburg, with 3 deaths, each of these fatal cases being very severe or complicated. A recurrence of haemorrhage took place in 8 per cent., as compared with 20 per cant. in 100 cases treated by the old method. Most were dismissed cured before the eighth week, and no unfavourable effects were produced.

Wirsing 8 reported 42 cases in 1906, 14 of which had had recent haematemesis. In one case haemorrhage recurred. In 27 of these patients the acidity of the stomach contents was estimated; it was found that during the treatment the amount and percentage of hydrochloric acid was diminished, the latter on the average from 0.14 to 0.11 per cent. Some cases did not show this, and yet progressed as well as the others.

The method of Senator, which has some similar features to that of Lenhartz, may be mentioned here. It consists of the administration of gelatine, butter, and cream from the beginning. He (see reference ${ }^{13}$ ) has reported 50 cases fed upon this diet, of whom 2 died, that is, 4 per cent.

Ewald 9 uses nutrient enemas for three days after a haemorrhage, and then gives milk, butter, and eggs, followed by other foods, as in Lenhartz's plan. He reported 34 cases in 1906. The results; however, appear to be inferior to those of the Lenhartz method. In 14 cases death occurred either after operation or immediately after haemorrhage, and there was a recurrence of haemorrhage in 7 per cent.

Ewald considers that the Lenhartz method cannot be justified on theoretical grounds and is not worth the risk. The above facts and figures, however, do not support this view, and, so far as data have been collected, it appears to be less risky than other methods of treatment.

Lenhartz in $1906^{10}$ reported 140 of his cases, all with recent haemorrhage by the mouth, or melaena. These include some of those reported by Wagner and Haberman. The mortality was 2.14 per cent. He recommends that in such cases great care should be taken not to increase the bulk of milk too rapidly, and in some instances quoted the eggs were given without the milk for two or three days. In oue case, by the twelfth day only $17 \mathrm{oz}$. of milk per day was being taken. The stom sch should never be distended. He reports that in two of his patients who died, on the fifteenth and eighteenth day respectively, the ulcer was found to be smooth and healed. He recommends the diet strongly for patients who have recently had a gastro-enterostomy performed.

Lambert 11 in 1907 published 5 severe cases, all of whom did well on the treatment. One was a woman of 32 years, who, after seven days' rectal feeding and seven days' careful feeding by the mouth with peptonized milk, still had occult blood in the stools and a haemoglobin percentage of 39, and the question of operation was being mooted. The Lenhartz treatment resulted in a cure. Another patient objected to the diet, even to the point of nausea and vomiting, but nevertheless, after the cessation of the diet for one day, did well. In another haemorrhage went on until the thirteenth day, and the case was regarded as below the safe limit for surgery, and yet made a good recovery on this treatment. A fourth case, with signs of peritoneal irritation, leucocytosis, and a temperature of $100^{\circ}$ to $104^{\circ}$, also recovered. In a fifth case, in which the haemoglobin was reduced to 20 per cent., in spite of an attack of enteric fever upon the twenty-second day, the treatment proved successful. Lambert's fourth case resembles one which came to my notice after the figures which I have given had been collected. A young woman of neurotic temperament was treated by $\mathbf{M r}$ G. E. Friend for gastric ulcer on the Lenhartz paln. She had no pain for four days, when vomiting recurred, with rigidity of the upper part of the abdomen. Perforation was discussed the patient was put upon enemata, and made a good recovery. It is a matter of conjecture whether she would or would not have recovered equally. well if, as in Lambert's case, the treatment had bien persisted in.

Berger, 12 in a publication from the Hamburg school, points out that so efficient has the method of treatment been found in the Eppendorfer Krankenhaus that it has proved useful as a method of diagnosis, for in cases which fail to respond the diagnosis has, in many instances, been at fault. He quotes six cases in which the failure of the diet led to the diagnosis of cancer, which was conflrmed by operation in four of them and in two by autopsy. In another case haemorrhage was found to arise from dilated veins about the oesophagus due to cirrhosis of the liver. He says, further, that in cases of pain without bleeding which do not yield, nervous diseases may be suspected.

Schnütgen ${ }^{18}$ mentions in 1907 that Lenhartz had then treated 201 patients, with a mortality of 3 per cent. This is about the same as the mortality in 195 cases treated by nutrient enemas by Leube, which was 4 per cent. Leube has altogether collected 556 cases, with a mortality of 2.2 per cent., but many of these were not of the hospital class.

It appears, therefore, that the death-rate of patients treated by the Lenhartz diet is not greater than that of those treated in other ways.

Finally, Dr. Langdon Brown ${ }^{14}$ in a recent paper mentions that he has treated 11 cases by the Lenhartz method with good results in 9 of them.

In conclusion, from an examination of these two series of cases, it may be said:

First, that the Lenhartz method of treatment is not more dangerous than treatment by nutrient and saline enemas followed by a graduated milk diet. In these particular cases the recurrence of haemorrhage was less frequent, and there were no deaths.

Secondly, that the pain suffered by the patient in the course of treatment is less on the Lenhartz diet.

Thirdly, the diet gives far more nourishment than can be introduced into the body by nutrient enemas, and is, therefore, more desirable in patients who have frequently been for a long time in a state of semi-starvation, or have suffered a loss of blood, or both.

Fourthly, that in cases treated by this method rectal injections may be entirely avoided. This is an advan. tage in a hospital, and a still greater advantage in treating cases at their homes, where rectal injections are not only regarded as extremely unpleasant, but are seldom efficiently administered.

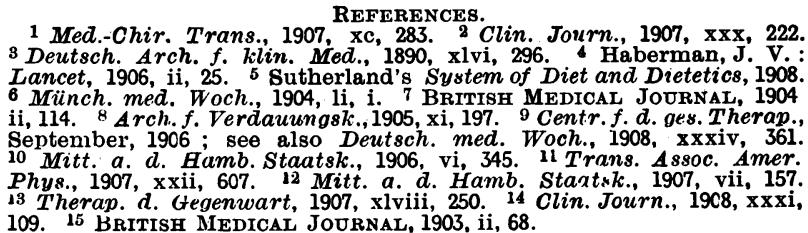
13 Therap. d. Gegenwart, 1907, xlviii, 250. 14 Clin. Journ., 19c8, xxxi, 109. 15 BRITISH Medical Journal, 1903, ii, 68.

\section{THE EARLY DIAGNOSIS AND TREATMENT}

\section{OF CANCER OF THE STOMACH.}

BY W. HALE WHITE, M.D., F.R.C.P., SENIOR PHYSICLAN TO GUY'S HOSPITAL.

There can be no doubt about the importance of this subject. The stomach is a very common seat of primary malignant disease; indeed, in nearly half of all the cases of carcinoma seen in the post-mortem room the primary seat of the disease is in the stomach, and although figures from the post-mortem room indicating the allocation of primary cancer to different organs cannot be accurately transferred to the living, for many cancers-for example, of the breast-which can be easily observed are excised early, yet the post-mortem figures show that cancer of the stomach is terribly frequent. It causes 1.5 per cent. of all deaths at Guy's Hospital. The necessity-if we are to do any long-lasting good to these patients-of an early diagnosis is obvious, for we know of no medical means of successfully treating cancer of the stomach, and with our present knowledge we know of no other proper treatment for cancer than excision, but my experience is that almost invariably by the time a patient comes into the hospital, or by the time a consultation is sought in private practice, the disease is too advanced for excision.

There are many cases in which an early diagnosis is impossible, for the patient does not seek advice until secondary deposits have developed. Thus it is not uncommon for the patient not to consult a doctor until he has secondary cancer of the liver, and in some such cases the most careful examination and investıgation of the history fails to suggest the seat of the primary growth. Still in some cases at least there might, I think, be some improvement in our early diagnosis.

The patient's history is of the greatest importance. Three-fourths of all the cases occur between the ages of 40 and 70 , and that in spite of there being far fewer people alive then than at earlier periods. There are an enormous number of persons who suffer from dyspepsia unassociated with any organic disease, but such usually have "symptoms of dyspepsia when they are young adults, therefore if symptoms of serious chronic gastric indigestion first appear after the age of 40, organic disease of the stomach should be strongly suspected, and if a comparatively short period of medical treatment does not effect a cure, it may be quite justifiable to open the abdomen to see if a growth is present, and, if possible, to take it away. In all cases of gastric carcinoma the uttermost endeavour must. be made to diagnose carcinoma of the stomach before the

* Remarks made in introducing a debate on the subject to which the paper relates at the Harveian Society of London. 
growth becomes tangible, for usually by such a time the period during which the growth could be excised has passed. There is no doubt that sometimes a simple ulcer of the stomach becomes cancerous; precisely how often this happens is very difficult to estimate. A simple ulcer becoming cancerous may occasionally be seen in the post-mortem room, but it is only reasonable to suppose that statistics from the deadhouse would underestimate the number of simple ulcers which became cancerous, for by the time of death the mass of growth is often so great that it is impossible to say whether or not a simple ulcer preceded it. On the other hand, clinical histories are very fallacious; often a history strongly suggestive of a previous gastric ulcer may be obtained from patients who have never had an ulcer. Nevertheless, Dr. W. J. Mayo, out of a large number of operations on the stomach, found that a malignant growth had appeared on an ulcer in about half the cases of gastric carcinoma, therefore a history of previous symptoms suggestive of ulcer of the stomach is a help to the early diagnosis of cancer of the stomach; but I have known a confident diagnosis of this disease made in a patient who gave a long history of ulcer, and who had a considerable mass to be felt in the region of the pylorus, but it turned out that no growth was present; the mass felt was the puckering and adhesion in connexion with a large ulcer. However, in these cases the ulcer itself may demand surgical treatment, and therefore opening the abdomen would be justified.

The commonest symptom of cancer of the stomach is pain, therefore we should be chary of diagnosing this disease if it is absent; on the other hand, constant pain is a considerable point in favour of such a diagnosis. Although the general health of the patient may be misleading, for sometimes it remains good, even when the growth has attained considerable size, yet usually those with cancer of the stomach feel more weak, are much wasted and are more anaemic than those whose dyspepsia is not associated with a growth. The red cells may fall to $3,000,000$, the colour index to 0.6 , and there may be a small increase in number of polymorphonuclear cells. Loss of appetite, nausea, and repugnance to food are usually well marked quite early in cancer of the stomach. On the other hand, these sym. ptoms are often absent when the indigestion is not due to growth. And we must always remember that the probability of cancer is greatly increased if any or all of these dyspeptic symptoms resist ordinary dietetic and other medical treatment.

Much attention has been directed to the amount of free hydrochloric acid present in the stomach of those suffering from gastric carcinoma. It is best after the fasting of the night to wash the stomach out with some water, then to give a test meal, such as a little bread and butter and milk, and an hour and a half after to siphon out the gastric contents. It appears that in a few cases of gastric carcinoma the free hydrochloric acid is not diminished, and it has been stated that this is especially likely to be the case when the carcinoma has developed on the site of an ulcer. Further, in many other gastric conditions, for example, atrophy of the gastric mucous membrane, and chronic gastritis, the hydrochloric acid may be diminished, although on the average not to the same extent as in gastric carcinoma. Carcinoma anywhere will diminish the amount of free hydrochloric acid - for example, in a case of carcinoma of the penis it was reduced to 0.00078 per cent. (the normal being 0.1951 per cent.). But the presence of carcinoma in other parts of the body is frequently easy of recognition. Therefore, in a case in which gastric carcinoma is suspected, the diminution of free hydrochloric acid is of help in diagnosis; but it must be a considerable diminution, for various diseases otker than malignant disease will diminish it somewhat. These results are the outcome of work by Moore, Morton Palmer, and others. The observations must be made by competent chemists, for it is not an easy matter to estimate the percentage of free hydrochloric acid in the gastric contents.

It is hardly necessary to mention that these should be carefully examined under the microscope, for occasionally cancer cells may be seen. Sometimes, too, the presence of a few blood discs or blood pigment, as shown by the guaiacum test, will be of help in diagnosis; but before relying on the guaiacum test we must be sure that the patient has not recently taken any meat. The occurrence of haemorrhage for the first time from the stomach in a person past the age of 40 , if he gives no bistory suggestive of ulcer, and bas not an obvious cause-such as cirrhosisfor his haemorrhage, is strongly suggestive of cancer. In 35 to 40 per cent. of all the cases of gastric carcinoma the vomit at some time or another contains blood visible to the naked eye.

In rather over 60 per cont. of the cases of carcinoma of the stomach the growth is at the pylorus, and in a considerable number of such cases there is a consequent obstruction to the exit of food from the stomach. Percussion without artificial distension is an uncertain means of estimating the size of the stomach, and a little succussion is fallacious; therefore these signs should not be relied upon in determining whether the patient has some gastric dilatation as a result of pyloric obstruction, but if a patient is given some bread and milk mixed with bismuth oxychloride and then examined in a vertical position by the $x$ rays with a screen, delay in the passage of the bismuth through the pylorus may readily be seen; and indeed probably the bismuth method may sometimes help us to discover a growth in other parts of the stomach, for the passage of the bismuth in a normal stomach is such a regular uniform motion that, if any of it adhered to the growth or this impaired the regular contraction of the stomach, we might see the unusual movement of the bismuth on the screen. An examination by the $x$ rays may be of great help in the diagnosis of cancer of the stomach, but too much reliance must not be placed upon it as a means of estimating whether the organ is dilated, for the position of the lower border of the stomach is very variable in health. Vomitjng large quantities at a time of sour material is evidence of gastric dilatation, but we ought to try to diagnose these cases before the quantity is excessively large. Lactic acid in the vomit indicates pyloric obstruction, for it shows that the gastric contents have remained in the stomach long enough to decompose, and this too is the significance of the Oppler-Boas bacillus. Visible peristaltic movements of the stomach, generally best elicited by flipping the abdominal wall, are a valuable sign, for, as they indicate hypertropby of the gastric muscle, if they are present we may often infer that the pylorus is obstructed. Dilatation of the stomach may, too, be made out by percussing the organ before and after the patient has swallowed a solution of bicarbonate of soda followed by some lemon juice. With regard to all these symptoms of dilatation of the stomach, it must be remem. bered that in order to diagnose gastric carcinoma from them we must show that pyloric obstruction is the cause of the dilatation; hence the value of the bismuth method and visible peristalsis.

Even when the growth cannot be removed, or secondary. deposits render this undesirable, much benefit often follows a gastro-enterostomy if there are signs of pyloric obstruction. The vomiting ceases; the patient digests bis food better and gains weight. Life will be prolonged partly for this reason, and probably because some of the gastric contents will no longer pass over the growth and hence it is less irritated and it will grow more slowly. It is, I think, clear that there are no means of infallibly diagnosing cancer of the stomach in its early stages; but I do believe that by carefully considering a number of points we might sometimes arrive at a diagnosis earlier than we do, and therefore give the patient the chance of excision, for medicines are powerless to cure him. Speaking generally, it is only in the early stages that excision can do any good, for secondary deposits usually form rapidly, and before subjecting the patient to an operation the whole body must be thoroughly examined to ascertain if any can be detected, and special attention must be paid to the liver and the left supraclavicular glands. Further, each individual case must most carefully be considered, for there is no doubt that many persons have had their lives shortened by excision of a gastric carcinoma; perhaps this, howerer, would not so often have been the case had the diagnosis been reached earlier. Lastly, evon if it is decided that excision' is impossible, consideration should be given to gastroenterostomy, for the relief which follows this when the pylorus is obstructed may be very great. 